\title{
Subchronic Administration of Linalool Decreases Depressive-Like Behaviour in Restrained Rats
}

\author{
Somrudee Saiyudthong ${ }^{1}$, Dawrung Srijittapong ${ }^{1}$ and Chantana Mekseepralard ${ }^{2}$ \\ 1. Department of Physiology, Faculty of Medicine, Srinakharinwirot University, Bangkok 10110, Thailand \\ 2. Department of Microbiology, Faculty of Medicine, Srinakharinwirot University, Bangkok 10110, Thailand
}

\begin{abstract}
Objectives: The aim of this study was to investigate the effect of linalool in chronically stressed rats on their behaviour as related to depressive disorders and BDNF (brain-derived neurotropic factor) protein in the hippocampus. Methods: Either Tween 80 or linalool $(50,160,500 \mathrm{mg} / \mathrm{kg})$ was intraperitoneally administered to rats, daily, for two weeks. Some rats were housed in home cages but the others were induced with chronic restrained stress (15 min daily). At the end of the treatment, the rats were assessed for depressive-like behaviour using the forced swimming test. At the end of the behaviour test, the animals were immediately decapitated and the hippocampus of each animal was removed for the measurement of the BDNF protein by ELISA. Result: The immobility time was significantly increased $(p<0.05)$ but time of climbing was significantly decreased $(p<0.05)$. The restrained rats treated with linalool, $500 \mathrm{mg} / \mathrm{kg}$, displayed immobility times less than those of their controls $(p<0.05)$ while these rats showed significantly more climbing than in the control rats $(p<0.05)$. Linalool showed no effect on the BDNF protein in the hippocampus. Conclusions: linalool decreases behaviour related to depressive disorders but it has no effect on the BDNF protein in chronic restrained stress.
\end{abstract}

Key words: Depression, linalool, force swimming test, BDNF, restrained stress.

\section{Introduction}

Chronic stressful events play an important role in the pathogenesis of depression. The relationship between depressive disorders and hyperactivation of the HPA (hypothalamo-pituitary-adrenocortical) axis has been widely established [1, 2]. The hyperactivation of the HPA axis induces depression-like behavior in the FST (forced swimming test), an animal model used for testing depression [3]. Chronic stress results in a decreased BDNF (brain-derived neurotropic factor) protein level in the hippocampus [4].

Antidepressant drugs have been widely prescribed for the treatment of depression. However, commonly found side effects include sleepiness during the day, dry mouth, profuse sweating, sexual dysfunction and constipation [5, 6]. Therefore, the development of effective complementary/alternative with less toxic effects has been needed. Aromatherapy, one of the

Corresponding author: Somrudee Saiyudthong, Ph.D., research fields: physiology. alternative treatments, has been used to relieve stress and depression for centuries. Many essential oils, used in aromatherapy, demonstrate antidepressant-like effects [7, 8]. Previous studies in mice have shown that the essential oil of Perilla frutescens attenuates the depressive-like behavior and reduces in both BDNF mRNA expression and protein level in the hippocampus $[9,10]$.

Linalool, a monoterpene compound, has been identified as a major component of many essential oils including lavender and bergamont. Linalool has shown antioxidant properties in several studies [11]. Inhalation of linalool induced sedation in mice without significant impairment in motor coordination, a side effect induced by most psycholeptic drugs [12]. A recent study has shown that linalool is a neuroprotective agent against acrylamide-induced neurotoxicity in rats [13]. The intraperitoneal injection of linalool significantly decreased the immobility time, an index of the depressive-like behaviour using the FST of mice as compared with the vehicle [14]. 
Besides, linalool has also shown an antidepressant-like activity in the TST (tail suspension test), another animal model used for evaluating antidepressant activity [15]. However, there has been no literature which reports about the antidepressant effects of linalool against stressful conditions.

The aim of the present study was to investigate the effects of linalool in chronically strained rats on their behaviour related to depressive disorders and BDNF protein level in the hippocampus.

\section{Materials and Methods}

\subsection{Animals}

Male Wistar rats, weighing 200-250 g, were purchased from the National Laboratory Animal Centre (Mahidol University, Bangkok, Thailand). The rats were housed, four per cage, at a constant temperature of $22 \pm 0.5{ }^{\circ} \mathrm{C}$ and under a $12: 12 \mathrm{~h}$ light/dark cycle. Standard laboratory food (National Laboratory Animal Center, Thailand) and tap water were available ad libitum. The rats, allowed to adapt to the new environment for one week, were inexperienced to the FST and naïve to exposure to essential oil or drugs. This research project was approved by the Animal Ethics Committee of Srinakharinwirot University (under license No. 13/2555), which is in compliance with the International Guiding Principles for Biomedical Research Involving Animals as provided by the National Research Council of Thailand.

\subsection{Chemicals}

Linalool (Sigma-Aldrich, St. Louis, USA) was diluted with Tween 80. Treatment groups were administrated with linalool 50, 160 and $500 \mathrm{mg} / \mathrm{kg}$, intraperitoneally. Based on the evidences from a previous $\mathrm{LD}_{50}$ study in rats has suggested that no death were observed at a single intraperitoneal injection of linalool 50, 160 and $500 \mathrm{mg} / \mathrm{kg}$ while all rats died after receiving 2 higher doses of linalool: $1600 \mathrm{mg} / \mathrm{kg}$ and $5000 \mathrm{mg} / \mathrm{kg}$. Therefore, we selected 50, 160 and 500 $\mathrm{mg} / \mathrm{kg}$ to examine depressive-like behavior [16]. Control groups were injected with Tween 80 alone.

\subsection{Experimental Design}

Control groups were put in either home cages or restrained stress tubes. All rats were divided into 5 groups ( $\mathrm{n}=10)$ : (1) Tween 80 + HC (home cage); (2) Tween 80 + RS (restrain stress); (3) linalool 50 mg/kg + RS; (4) linalool 160 mg/kg + RS and; (5) linalool $500 \mathrm{mg} / \mathrm{kg}+\mathrm{RS}$. Either linalool or Tween 80 was intraperitoneally administered daily for two weeks. At the end of the treatment, the rats were evaluated for depressive-like behaviour using the FST between the hours of 0900 and 1200. Following the behavioral assessments, the rats were immediately decapitated and the hippocampus of each rat was dissected, weighed and stored at $-80^{\circ} \mathrm{C}$ until an assay for the BDNF protein was done (Fig. 1).

\subsection{Restrained Stress}

To induce stress, the restraints were performed in an adjustable restraint tube $(15.5 \pm 2.5 \mathrm{~cm}$ long and $6.3 \mathrm{~cm}$ diameter) with ample holes for ventilation. Rats were immobilized in the restraint for 15 min stress sessions daily between the hours of 0900-1200. The restraining was continued for two weeks. The immobilization procedure is considered to be a psychological stress or as it does not produce pain or direct physical insult [17]. At the end of each daily stress, each rat was returned to their cages.

\subsection{Forced Swimming Test (FST)}

The FST, known as the Porsolt test, was performed for screening antidepressants [18]. The forced swimming tank consists of a clear, cylindrical tank (40 $\mathrm{cm}$ height $\times 18 \mathrm{~cm}$ diameter), containing tap water at a depth of $30 \mathrm{~cm}$ maintained at a temperature of $25{ }^{\circ} \mathrm{C}$. The FST consisted of 2 trials, conditioning and testing. During the conditioning trial, the rats were individually placed into the forced swimming tank for $15 \mathrm{~min}$. The rats were returned to their home cages after they were 


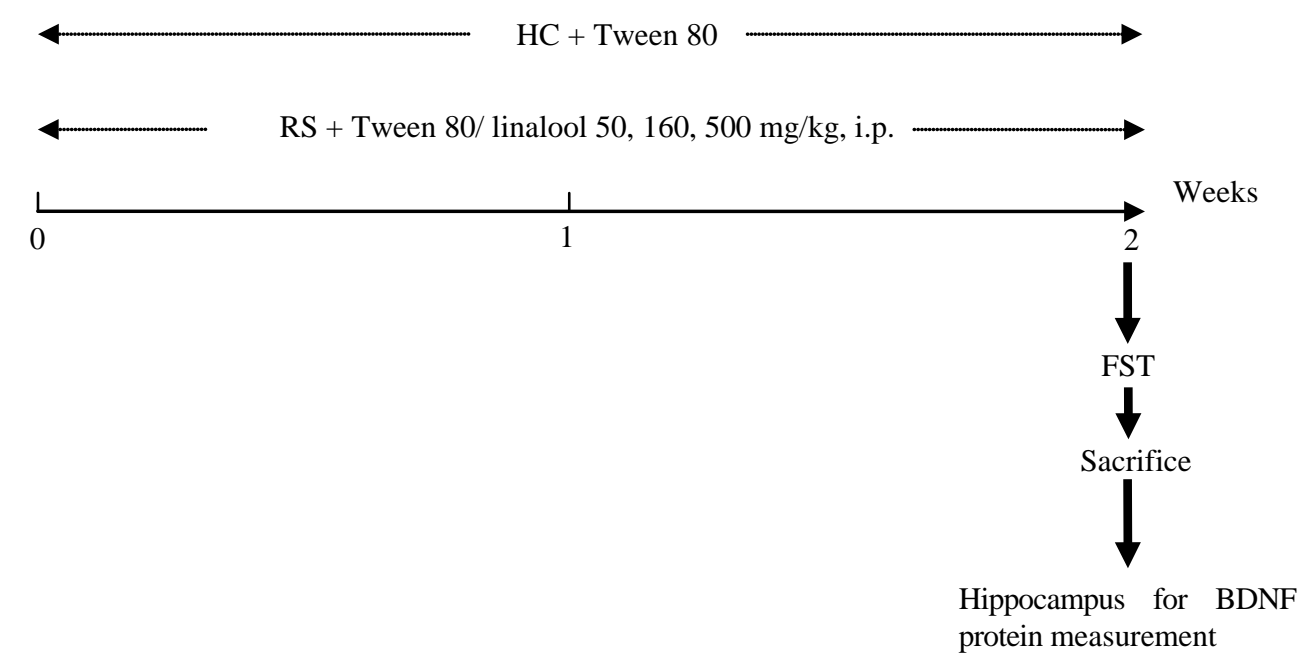

Fig. 1 Experimental timeline. Control groups were put in either home cages or restrained stress tubes for two weeks. Either linalool (50, 160, $500 \mathrm{mg} / \mathrm{kg})$ or Tween 80 was intraperitoneally administered daily for an entire two weeks. At the end of the treatment, rats were evaluated for depressive-like behavior using the FST. Then, the rats were immediately decapitated and the hippocampus was removed for the measurement of the BDNF protein.

dry. The test trial was carried out 24 hours later. Each rat was left in the swimming tank for $6 \mathrm{~min}$. After the first $2 \mathrm{~min}$, the immobility time (floating passively and keeping their head above the water) was measured during the last 4 min of the test. The times of climbing the basin wall and active swimming were also recorded. During the test session, three behavioural categories were recorded with a videotape for subsequent analysis.

\subsection{Measurement of BDNF Protein Levels}

Rat BDNF protein levels in the hippocampus were determined in an antibody sandwich format using the BDNF $E_{\max }{ }^{\circledR} \quad$ Immunoassay system (Promega Coporation, USA) as described in the manufacturer's instructions. Briefly, the hippocampus was homogenized in a RIPA buffer (Sigma-Aldrich, St. Louis, USA) containing 2\% BSA, 5\% glycerol and 10\% protease inhibitor. Each homogenate was left on ice for $10 \mathrm{~min}$ and centrifuged at $14,000 \mathrm{~g}$ for $60 \mathrm{~min}$. The supernatant of the hippocampus homogenate was then stored at $-80^{\circ} \mathrm{C}$ until use. A flat-bottom 96-well ELISA plate was coated with anti-BDNF monoclonal antibody and incubated at $4^{\circ} \mathrm{C}$ overnight. After blocking the non-specific binding, each hippocampus homogenate supernatant was diluted at 1:4. A 1:2 serial dilution of BDNF standard protein was added in the wells and incubated at room temperature for 2 hours. The captured BDNF proteins were detected by incubating with a polyclonal antibody against BDNF followed by anti-IgY antibody conjugated to horseradish peroxidase at room temperature for 2 hours and 1 hour, respectively. A TMB one solution was used as a chromogenic substrate and the reaction was stopped with $1 \mathrm{~N}$ hydrochloric acid. This procedure was repeated in triplicate for each rat and the concentrations of the BDNF protein were calculated from a BDNF standard curve.

\subsection{Statistical Analysis}

All data are expressed as the mean \pm SEM. The data were analyzed by one-way ANOVA with a post hoc Dunnett's test on the GraphPad Prism. The differences were considered statistically significant when $p<0.05$.

\section{Results}

In chronic restrained rats tested in the FST, the immobility time was significantly increased but the time of climbing was significantly decreased $(p<0.05)$. Restrained rats treated with linalool $500 \mathrm{mg} / \mathrm{kg}$ 
exhibited immobility times less than their controls $(p<$ 0.05 ) while they showed significantly more climbing than seen in the control rats. No differences were found in the time the rats spent for active swimming (Fig. 2). Furthermore, either restrained stress or linalool had no effects on the levels of the BDNF protein (Fig. 3).

A

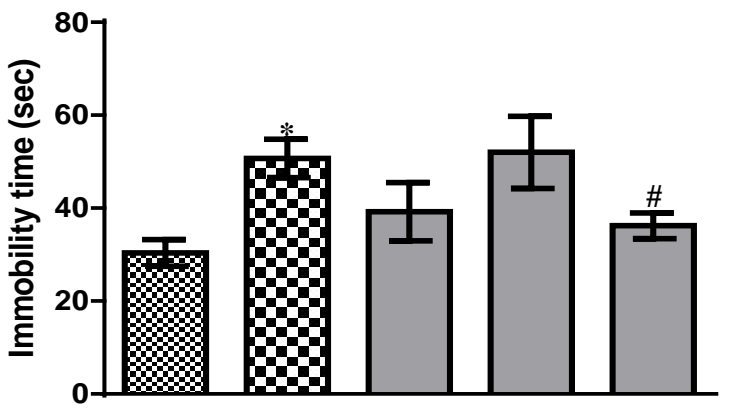

B

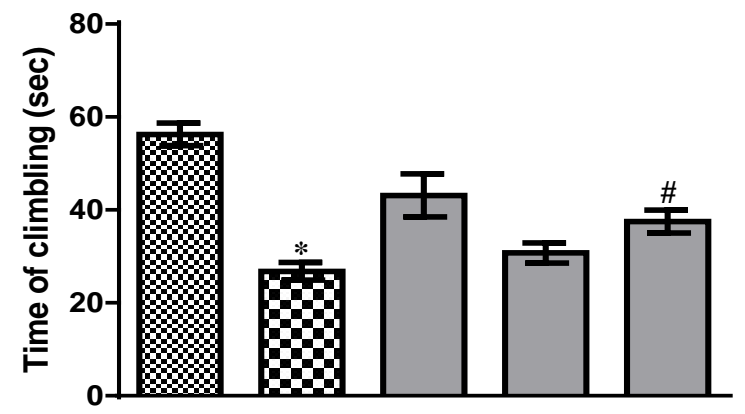

C

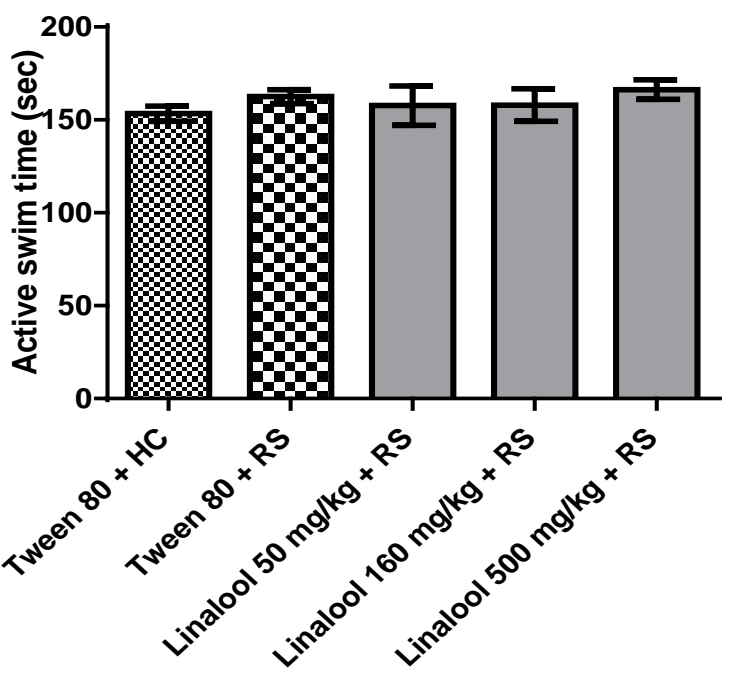

Fig. 2 Effects of subchronic linalool treatment on the forced swimming test. Behavior parameters (immobility time, time of climbing and active swimming time) of rats, housed in HC (home cages), were compared with RS (restrained stress). Linalool (50, 160, $500 \mathrm{mg} / \mathrm{kg}$, i.p.) was compared to controls (Tween 80, i.p.) in restrained stress conditions. Data are expressed as mean \pm SEM $(n=10) . * p<0.05$ compared to Tween $80+$ HC, \# $p<0.05$ compared to Tween $80+$ RS. 


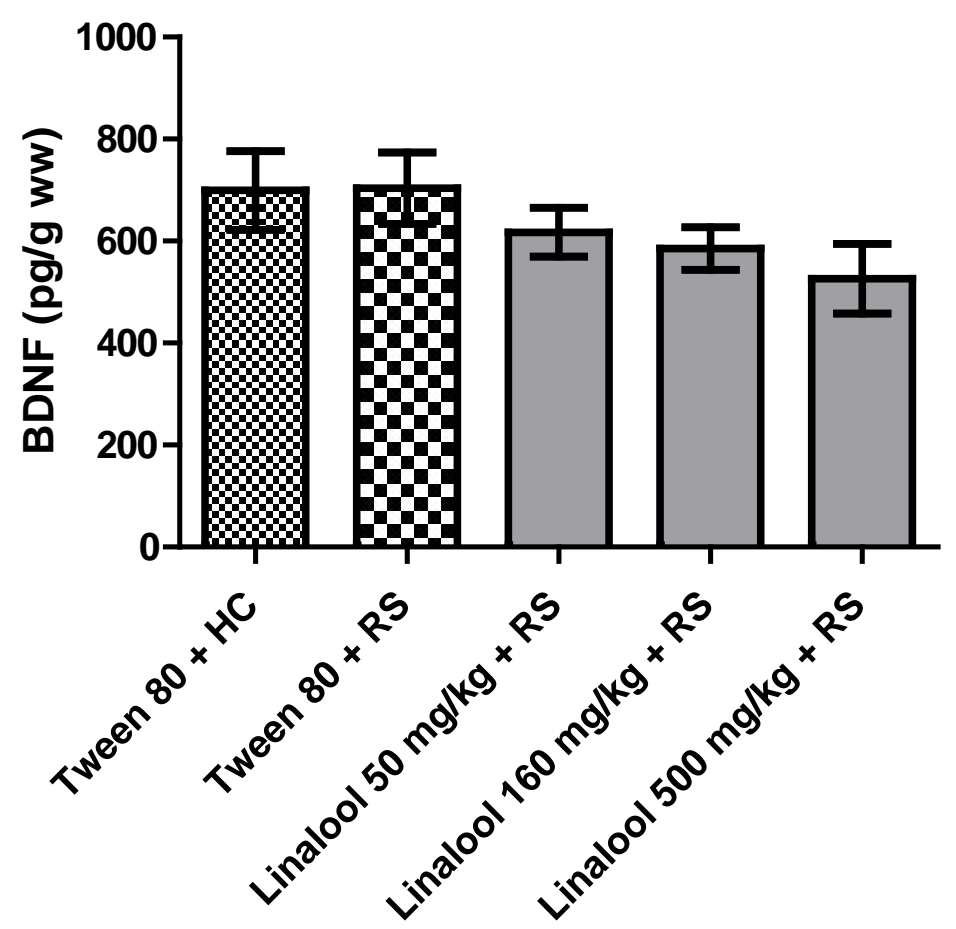

Fig. 3 Effect of subchronic linalool treatment on BDNF (brain-derived neurotrophic factor). BDNF protein level of rats, housed in HC (home cages), was compared with that of rats with RS (restrained stress). Linalool (50, 160, $500 \mathrm{mg} / \mathrm{kg}$, i.p.) was compared to controls (Tween 80, i.p.) in restrained stress conditions. Data are expressed as mean \pm SEM (n = 10).

\section{Discussion}

The results of the present study demonstrate that sub chronic treatment with linalool decreases behaviour related depressive disorder but has no effect on the BDNF protein in chronic restrained stress.

The FST, behavioural despair test or Porsolt test has been used to screen new antidepressants [18]. The duration of the immobility was mostly regarded to be an index of the depressive-like behaviour in animals such as rats and mice. Moreover, the time of active swimming and climbing in the FST were also investigated. Chronic treatment with antidepressant drugs, including fluoxetine, increased swimming times but decreased immobility times [19, 20]. Administration of fluoxetine to Swiss mice significantly decreased the duration of immobility time but increased the duration of climbing [21]. The present study showed that the injection with linalool $500 \mathrm{mg} / \mathrm{kg}$ in stressed rats for two weeks reduced immobility times but increased climbing times when compared to their controls suggesting that this dose produced antidepressant activity. Our results are consistent with a previous study demonstrating that the acute administration of linalool showing antidepressant-like activity in the FST on mice [14].

The mechanism by which linalool exhibits an antidepressant-like activity in long-term treatment remains unknown though current evidence suggests that linalool produces an antidepressant-like effect through interaction with the monoaminergic system, including serotonergic and noradrenergic systems. A previous study has shown that the acute treatment with linalool has demonstrated an antidepressant-like effect involving the serotonergic pathway through postsynaptic 5- $\mathrm{HT}_{1 \mathrm{~A}}$ receptors [22]. Besides, pre-treatment with yohimbine (a $\alpha_{2}$-receptor antagonist) modified the antidepressant-like effects of linalool on the FST, suggesting the involvement of the noradrenergic system. Future studies are needed to investigate 
whether the antidepressant effects of linalool after long-term treatment also involves the serotonergic and noradrenergic systems.

BDNF plays a major role in the regulation of neurogenesis during development and the maintenance of adult neurons. Previous animal studies have shown that exposure to chronic stress results in the decrease of BDNF mRNA/protein levels in the hippocampus [11, $23,24]$. However, in the present study, BDNF protein levels did not show any reduction following the chronic restrained stress (15 min exposure) although this duration of restrained stress has shown the highest corticosterone release in a previous study [17]. The result is well agreed with a previous study that has shown that BDNF and its receptor, TrkB (tyrosine kinase receptor $\mathrm{B}$ ), expression are decreased in the 3 hours stress group but not in the 1 hour stress group when compared to the control group. This suggests that the alteration in BDNF protein level is dependent on the duration of restraint stress. In addition, subchronic treatment with antidepressants, including fluoxetine and reboxetin, reversed the decrease of BDNF protein induced by chronic stress in the rat hippocampus [4, 25]. However, the present study has shown that subchronic linalool treatment (daily i.p. for two weeks) had no effect on BDNF levels in the hippocampus. The reason for these results may be due to the shorter time course of the treatment used in this study than that of previous studies. Therefore, the next study should extend the treatment period before concluding the role of linalool on BDNF level.

\section{Conclusions}

The present study demonstrates that subchronic administration of linalool decreases depressive-like behavior in restrainted stress in rats. However, BDNF levels in the hippocampus did not show any changes after 2-weeks of treatment with linalool. Therefore, longer periods of treatment with linalool are needed to be investigated the intracellular mechanism.

\section{Acknowledgments}

This research was supported by a grant from Srinakharinwirot University (130/2555). Our thanks go to Mrs. Chonticha Areebambud for her technical assistance.

\section{Conflict of Interest}

The authors declare that they have no conflicts of interest.

\section{References}

[1] Himmerich, H., et al. 2007. "Changes in the Hypothalamic-Pituitary-Adrenal Axis and Leptin Levels during Antidepressant Treatment." Neuropsychobiology 55: 28-35.

[2] Nikisch, G., et al. 2005. "Long-term Citalopram Administration Reduces Responsiveness of HPA Axis in Patients with Major Depression: Relationship with S-Citalopram Concentrations in Plasma and Cerebrospinal Fluid (CSF) and Clinical Response.” Psychopharmacology 181: 751-60.

[3] Murray, F., et al. 2008. "Chronic Low Dose Corticosterone Exposure Decreased Hippocampal Cell Proliferation, Volume and Induced Anxiety and Depression like Behaviors in Mice.” Eur J Pharmacol 583: 115-27.

[4] Filho, C. B., et al. 2015. "Chronic Unpredictable Mild Stress Decreases BDNF and NGF Levels and $\mathrm{Na}(+)$, $\mathrm{K}(+)$-ATPase Activity in the Hippocampus and Prefrontal Cortex of Mice: Antidepressant Effect of Chrysin.” Neuroscience 289: 367-80.

[5] Bet, P. M., et al. "Side Effects of Antidepressants during Long-term Use in A Naturalistic Setting." Eur Neuropsychopharmacol 23: 1443-51.

[6] Segraves, R. T., and Balon, R. 2014. “Antidepressant-induced Sexual Dysfunction in Men.” Pharmacol Biochem Behavior 121: 132-7.

[7] Cioanca, O., et al. 2014. "Inhalation of Coriander Volatile Oil Increased Anxiolytic-Antidepressant-like Behaviors and Decreased Oxidative Status in Beta-Amyloid (1-42) Rat Model of Alzheimer's Disease.” Physiol Behav 131: 68-74.

[8] Fißler, M., and Quante, A. 2014. “A Case Series on the Use of Lavendula Oil Capsules in Patients Suffering from Major Depressive Disorder and Symptoms of Psychomotor Agitation, Insomnia and Anxiety." Complement Ther Med 22: 63-9. 
[9] Ji, W. W., et al. 2014. “Antidepressant-like Effect of Essential Oil of Perilla Frutescens in a Chronic, Unpredictable, Mild Stress-induced Depression Model Mice.” Chin J Nat Med 12: 753-9.

[10] Yi, L. T., et al. 2013. "Essential Oil of Perilla Frutescens-induced Change in Hippocampal Expression of Brain-derived Neurotrophic Factor in Chronic Unpredictable Mild Stress in Mice.” J Ethnopharmacol 147: 245-53.

[11] Cieślik, K., et al. 2011. "Chronic Unpredictable Stress-Induced Reduction in the Hippocampal Brain-Derived Neurotrophic Factor (BDNF) Gene Expression is Antagonized by Zinc Treatment.” Pharmacol Rep 63: 537-43.

[12] Linck, V. M., et al. 2009. "Inhaled Linalool-induced Sedation in Mice.” Phytomedicine 16: 303-7.

[13] Mehri, S., et al. 2014. "Linalool as a Neuroprotective Agent against Acrylamide-Induced Neurotoxicity in Wistar Rats.” Drug Chem Toxicol 21: 1-5.

[14] Guzmán-Gutiérrez, S. L., et al. 2012. “Antidepressant Activity of Litsea Glaucescens Essential Oil: Identification of B-Pinene and Linalool as Active Principles.” J Ethnopharmacol 143: 673-9.

[15] Coelho, V., et al. 2013. "Neurobehavioral and Genotoxic Evaluation of (-)-Linalool in Mice.” $J$ Nat Med 67: 876-80.

[16] RIFM (Research Institute for Fragrance Materials Inc.). 1984. “A 14-Day Single Dose Subacute Toxicity Study in the Rat.” Unpublished Report from Lorillard Tobacco Company, 12 January. Report number 36660. (RIFM,
Woodcliff Lake, NJ, USA).

[17] Weinberg, M. S., et al. 2007. "Restraint-Induced Fra-2 and C-Fos Expression in the Rat Forebrain: Relationship to Stress Duration.” Neuroscience 150: 478-86.

[18] Porsolt, R. D., et al. 1987. "Behavioural Despair in Rats: A New Model Sensitive to Antidepressant Treatments.” Eur J Pharmacol 47: 379-91.

[19] Dulawa, S. C., et al. 2004. "Effects of Chronic Fluoxetine in Animal Models of Anxiety and Depression.” Neuropsychopharmacology, 29: 1321-30.

[20] Shumake, J., et al. 2010. "Metabolic Mapping of the Effects of the Antidepressant Fluoxetine on the Brains of Congenitally Helpless Rats.” Brain Res 1343: 218-25.

[21] Costa, A. P., et al. 2013. "A Proposal for Refining the Forced Swim Test in Swiss Mice.” Prog Neuropsychopharmacol Biol Psych 45: 150-5.

[22] Guzmán-Gutiérrez, S. L., et al. 2015. "Linalool and B-Pinene Exert Their Antidepressant-like Activity through the Monoaminergic Pathway.” Life Sciences 128: 24-29.

[23] Alboni, S., et al. 2001. "Stress Induces Altered CRE/CREB Pathway Activity and BDNF Expression in the Hippocampus of Glucocorticoid Receptor-Impaired Mice.” Neuropharmacology 60: 1337-46.

[24] Macedo, I. C., et al. 2015. “Chronic stress associated with hypercaloric diet changes the hippocampal BDNF levels in male Wistar rats.” Neuropeptides 51: 75-81.

[25] First, M., et al. 2013. "The Effects of Reboxetine Treatment on Depression-like Behavior, Brain Neurotrophins, and ERK Expression in Rats Exposed to Chronic Mild Stress.” J Mol Neurosci 50: 88-97. 